\title{
Spectral evolution of star clusters in the Large Magellanic Cloud
}

\section{Blue concentrated clusters in the age range $40-300 \mathrm{Myr}^{\star}$}

\author{
J. F. C. Santos Jr. ${ }^{1}$, J. J. Clariá ${ }^{2, \star \star}$, A. V. Ahumada ${ }^{2, \star \star}$, E. Bica ${ }^{3}$, A. E. Piatti ${ }^{4}$, and M. C. Parisi ${ }^{2, \star \star ~}$ \\ 1 Departamento de Física, ICEx, UFMG, CP 702, 30123-970, Belo Horizonte, MG, Brazil \\ e-mail: jsantos@fisica.ufmg.br \\ 2 Observatorio Astronómico, Laprida 854, 5000, Córdoba, Argentina \\ 3 Depto. de Astronomia, UFRGS, CP 15051, 91500-970, Porto Alegre, Brazil \\ ${ }^{4}$ Instituto de Astronomía y Física del Espacio, CC 67, Suc. 28, 1428, Buenos Aires, Argentina
}

Received 4 October 2005 / Accepted 15 November 2005

\section{ABSTRACT}

\begin{abstract}
Aims. Integrated spectroscopy of a sample of 17 blue concentrated Large Magellanic Cloud (LMC) clusters is presented and its spectral evolution studied. The spectra span the range $\approx(3600-6800) \AA$ with a resolution of $\approx 14 \AA$ FWHM, being used to determine cluster ages and, in connection with their spatial distribution, to explore the LMC structure and cluster formation history.

Methods. Cluster reddening values were estimated by interpolation, using the available extinction maps. We used two methods to derive cluster ages: (i) template matching, in which line strengths and continuum distribution of the cluster spectra were compared and matched to those of template clusters with known astrophysical properties, and (ii) equivalent width (EW) method, in which new age/metallicity calibrations were used together with diagnostic diagrams involving the sum of EWs of selected spectral lines (K Ca II, G band $(\mathrm{CH}), \mathrm{Mg} \mathrm{I}, \mathrm{H} \delta, \mathrm{H} \gamma$ and $\mathrm{H} \beta$ ).

Results. The derived cluster ages range from $40 \mathrm{Myr}$ (NGC 2130 and SL 237) to $300 \mathrm{Myr}$ (NGC 1932 and SL 709), a good agreement between the results of the two methods being obtained. Combining the present sample with additional ones indicates that cluster deprojected distances from the LMC center are related to age in the sense that inner clusters tend to be younger.

Conclusions. Spectral libraries of star clusters are useful datasets for spectral classifications and extraction of parameter information for target star clusters and galaxies. The present cluster sample complements previous ones, in an effort to gather a spectral library with several clusters per age bin.
\end{abstract}

Key words. galaxies: star clusters - Magellanic Clouds - techniques: spectroscopic

\section{Introduction}

Star clusters in the Magellanic Clouds can help us to have a better insight about the star formation history of the galaxies as a whole (Mighell et al. 1998; Piatti et al. 2001, 2002a). In particular, the large number of young and intermediate-age star clusters in the Large Magellanic Cloud (LMC) composes an ensemble which allows to probe the galaxy structure and evolution during the last few Gyrs. Indeed, the total estimated number of clusters in the LMC is $\approx 4200$ (Hodge 1988), which compares to the catalogued 4089 clusters, associations with characteristics of somewhat looser clusters and newly-formed ones (Bica et al. 1999). Episodes of enhancement or reduction in cluster

^ Figures 7 to 22 are only available in electronic form at http://www . edpsciences.org

$\star \star$ Visiting Astronomer, Complejo Astronómico El Leoncito operated under agreement between the Consejo Nacional de Investigaciones Científicas y Técnicas de la República Argentina and the National Universities of La Plata, Córdoba and San Juan. formation can be traced back in the galaxy's history if statistically meaningful samples of clusters with well-determined properties are considered.

The analysis of continuum and absorption lines of the integrated spectra of star clusters has provided accurate estimates of their ages and metallicities. The determination of these properties for distant and/or compact clusters, in which individual stars are not observable, needs a calibration based on clusters of well-known ages and metallicities. These age and metallicity calibrations are established by means of equivalent width (EW) measurements, in particular for conspicuous lines of $\mathrm{H}$, $\mathrm{Fe}$ and $\mathrm{Mg}$ in the visible spectra. Based on these data it is possible to characterize clusters of unknown properties as well as to use the integrated spectra as reference populations (templates) to investigate more complex stellar systems. In order to accomplish these two aims, integrated spectra of star clusters in the Magellanic Clouds (e.g. Bica et al. 1990; Santos et al. 1995; Piatti et al. 2005) and in the Galaxy (e.g. Santos \& Bica 1993; Piatti et al. 2002b) have been observed, catalogued 
(Santos et al. 2002), and analyzed (Santos \& Piatti 2004, hereafter SP).

The objective of the present work is to derive age for a sample of 17 LMC clusters, all of type II in the sequence defined by Searle et al. (1980, hereafter SWB), which corresponds to the age range (30-70 Myr) according to posterior photometric calibrations (e.g. Bica et al. 1996). The compact nature and high surface brightness of the present sample clusters make them good targets for integrated spectroscopy. For such young clusters we derived ages but it was not possible to derive metallicities using the proposed methods. Even though, cluster ages and deprojected positions provide useful information on the LMC properties. When our age estimates are combined with a larger sample (Piatti et al. 2003b) with ages in the same scale as in the present work, the interpretation of the data regarding the LMC structure and cluster formation history is improved.

The present cluster sample complements previous ones, in an attempt to provide a spectral library with several clusters per age bin. At the same time, we study the clusters themselves individually, determining their ages and analyzing their spatial distribution, in order to explore the LMC structure and cluster formation history. To estimate the clusters' ages, we employ the new calibrations and diagnostic diagrams recently provided by SP for visible integrated spectra, along with template spectra (e.g. Santos et al. 1995; Ahumada et al. 2002). The first method is based on the EW of Balmer $\mathrm{H}, \mathrm{Mg}$ and $\mathrm{Fe}$ lines and the second one on the continuum distribution of clusters with wellknown properties. We confirm the reliability of the procedure proposed by SP to determine clusters' ages, since we included in the sample not only unstudied or poorly studied clusters, but also the cluster NGC 1839 which has been previously observed by means of Washington photometry (Piatti et al. 2003a).

\section{Observations}

The observations were carried out at the Complejo Astronómico El Leoncito (CASLEO, San Juan, Argentina) with the $2.15 \mathrm{~m}$ telescope during four nights in December 2003. We employed a CCD camera containing a Tektroniks chip of $1024 \times 1024$ pixels attached to a REOSC spectrograph, the size of each pixel being $24 \mu \mathrm{m} \times 24 \mu \mathrm{m}$; one pixel corresponds to 0.94 " on the sky. The slit was set in the East-West direction and the observations were performed by scanning the slit across the objects in the North-South direction in order to get a proper sampling of cluster stars. The long slit corresponding to $4.7^{\prime}$ on the sky, allowed us to sample regions of the background sky. We used a grating of 300 grooves $\mathrm{mm}^{-1}$, producing an average dispersion in the observed region of $\approx 140 \AA / \mathrm{mm}$ (3.46 $\AA /$ pixel). The spectral coverage was $\approx(3600-6800) \AA$. The slit width was $4.2^{\prime \prime}$, providing a resolution (FWHM) of $\approx 14 \AA$, as deduced from the comparison lamp lines.

The reduction of the spectra was carried out with the IRAF $^{1}$ package at the Observatorio Astronómico de Córdoba

${ }^{1}$ IRAF is distributed by the National Optical Astronomy Observatories, which is operated by the Association of Universities for Research in Astronomy, Inc., under contract with the National Science Foundation.
(Argentina) following the standard procedures. Summing up, we subtracted the bias and used flat-field frames previously combined to correct the frames for high and low spatial frequency variations. We also checked the instrumental signature with the acquisition of dark frames. Then, we performed the background sky subtraction using pixel rows from the same frame, after having cleaned the background sky regions from cosmic rays. We controlled that no significant background sky residuals were present on the resulting spectra. The cluster spectra, which were extracted along the slit according to the cluster size and available flux, were then wavelength calibrated by fitting observed $\mathrm{Cu}-\mathrm{Ar}-\mathrm{Ne}$ comparison lamp spectra with template spectra. The rms errors involved in these calibrations are on average $0.40 \AA$ A Finally, extinction correction and flux calibrations derived from the observed standard stars were applied to the cluster spectra. In addition, cosmic rays on them were eliminated. Table 1 presents the cluster sample including the designations in different catalogues, the equatorial coordinates, the average diameters according to Bica et al. (1999) and the averaged signal-to-noise $(\mathrm{S} / \mathrm{N})$ ratios of the spectra. Typically, three spectra were obtained per cluster.

A colour-colour diagram of the sample is presented in Fig. 1, where the clusters integrated UBV colours are situated among those from a larger sample (Bica et al. 1996) characterizing the whole age range of LMC clusters.

\section{Analysis of the cluster spectra}

Cluster ages were derived by means of two methods: the template matching method, in which the observed spectra are compared and matched to template spectra with welldetermined properties (e.g. Piatti et al. 2002b, and references therein), and the EW method, in which diagnostic diagrams involving the sum of EWs of selected spectral lines were employed together with their calibrations with age and metallicity (SP). In the first method, a high weight is assigned to the overall continuum, while in the second method the spectral lines are the relevant observables. Both methods rely on the library of star cluster integrated spectra with well-determined properties, accomplished in various studies (e.g. Bica \& Alloin 1986; Piatti et al. 2002b, and references therein) and made available through the CDS/Vizier catalogue database at http://vizier.u-strasbg.fr/cgi-bin/ VizieR?-source=III/219 (Santos et al. 2002).

\subsection{Equivalent width method}

Before measuring EWs in the observed spectra, these were set to the rest-frame according to the Doppler shift of H Balmer lines. Next, the spectra were normalized at approximately $5870 \AA$. The flux normalization at this wavelength is meant to represent the continuum flux around $5870 \AA$, avoiding spectral lines eventually present. In practice, the spectral region around $5870 \AA$ ( $\approx 20 \AA$ wide) is examined and the normalization applied to a nearby wavelength that is representative of the continuum flux.

Spectral fluxes at $3860 \AA, 4020 \AA, 4150 \AA, 4570 \AA$, $4834 \AA, 4914 \AA$ and $6630 \AA$ were used as guidelines to 
Table 1. The sample clusters.

\begin{tabular}{|c|c|c|c|c|}
\hline Name $^{a}$ & $\begin{array}{c}\alpha_{2000} \\
(\mathrm{~h}: \mathrm{m}: \mathrm{s})\end{array}$ & $\begin{array}{c}\delta_{2000} \\
\left({ }^{\circ}:^{\prime \prime}:^{\prime \prime}\right)\end{array}$ & $\begin{array}{l}\mathrm{D}^{b} \\
\left({ }^{\prime}\right)\end{array}$ & $S / N$ \\
\hline NGC 1804, SL 172, ESO 56-SC 46, LMC_OGLE 8 & 5:01:04 & $-69: 04: 57$ & 0.90 & 35 \\
\hline NGC 1839, SL 226, ESO 56-SC 63, LMC_OGLE 93 & 5:06:02 & $-68: 37: 36$ & 1.60 & 50 \\
\hline SL 237, LMC_OGLE 116 & 5:06:57 & $-69: 08: 51$ & 1.00 & 30 \\
\hline NGC 1870, SL 317, ESO 56-SC 81, LMC_OGLE 235 & 5:13:10 & $-69: 07: 01$ & 1.05 & 95 \\
\hline NGC 1894, SL 344, ESO 56-SC 89, BRHT 8a, LMC_OGLE 278 & 5:15:51 & $-69: 28: 09$ & 1.30 & 45 \\
\hline NGC 1902, SL 367, ESO 85-SC 66, KMHK 734 & $5: 18: 18$ & $-66: 37: 38$ & 1.70 & 35 \\
\hline NGC 1913, SL 373, ESO 56-SC 97, BRHT 10a, LMC_OGLE 330 & $5: 18: 21$ & $-69: 32: 31$ & 1.20 & 25 \\
\hline NGC 1932, SL 420, ESO 85-SC 77, KMHK 825 & $5: 22: 27$ & $-66: 09: 09$ & 1.30 & 30 \\
\hline NGC 1943, SL 430, ESO 56-SC 114, LMC_OGLE 411 & $5: 22: 29$ & $-70: 09: 17$ & 1.05 & 30 \\
\hline NGC 1940, SL 427, ESO 85-SC 78, KMHK 834 & $5: 22: 43$ & $-67: 11: 10$ & 1.20 & 35 \\
\hline NGC 1971, SL 481, ESO 56-SC 128, BRHT 12a, LMC_OGLE 480 & $5: 26: 45$ & $-69: 51: 07$ & 1.03 & 50 \\
\hline SL 508, LMC_OGLE 518 & 5:29:20 & $-69: 35: 56$ & 1.00 & 45 \\
\hline NGC 2038, SL 590, ESO 56-SC 158, KMHK 1110, LMC_OGLE 607 & $5: 34: 41$ & $-70: 33: 39$ & 1.55 & 45 \\
\hline SL 709, BM 63, KMHK 1350 & $5: 46: 15$ & $-67: 34: 06$ & 1.03 & 25 \\
\hline NGC 2118, SL 717, ESO 57-SC 39, KMHK 1380 & $5: 47: 39$ & $-69: 07: 54$ & 1.60 & 60 \\
\hline NGC 2130, SL 758, ESO 86-SC 37, KMHK 1476 & 5:52:23 & $-67: 20: 02$ & 1.35 & 25 \\
\hline NGC 2135, SL 765, ESO 86-SC 39, BM 151, KMHK 1496 & $5: 53: 35$ & $-67: 25: 40$ & 1.50 & 20 \\
\hline
\end{tabular}

${ }^{a}$ Cluster identifications are from Lauberts (1982, ESO), Shapley \& Lindsay (1963, SL), Bhatia et al. (1991, BRHT), Kontizas et al. (1990, KMHK), Bhatia \& MacGillivray (1989, BM), Pietrzyński et al. (1999, LMC_OGLE).

${ }^{b}$ Average diameter according to Bica et al. (1999).
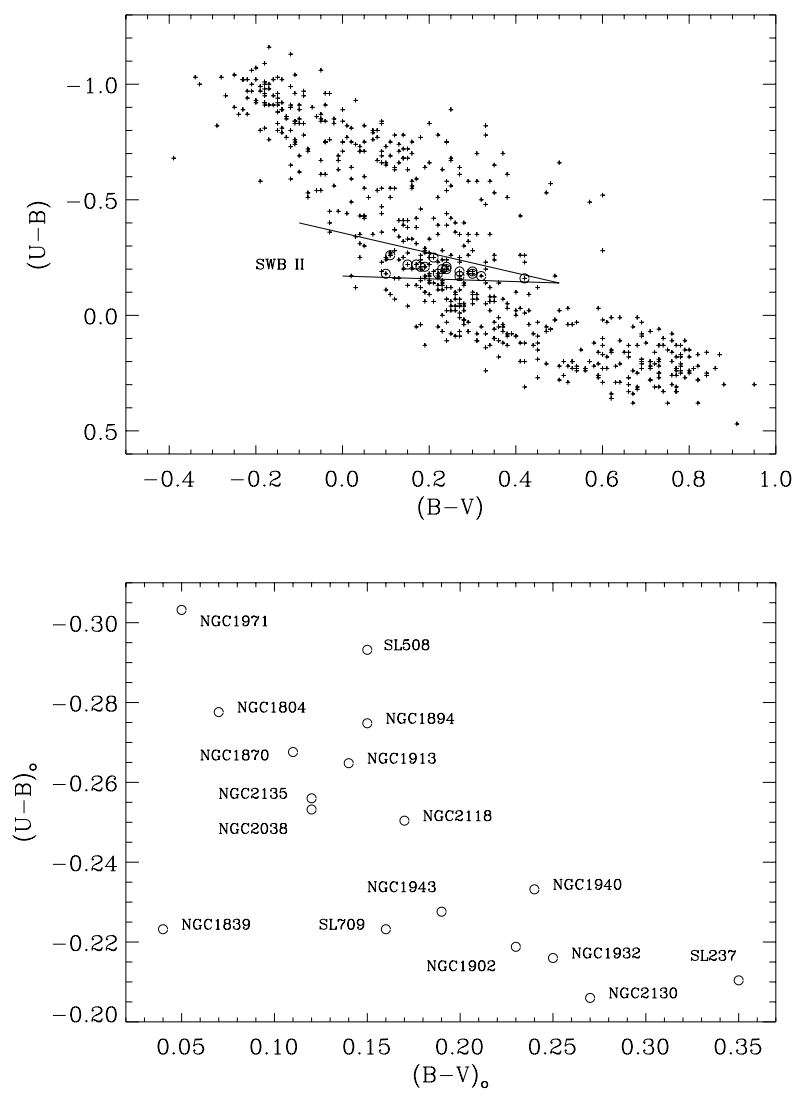

Fig. 1. Colour-colour diagrams of the 17 clusters (open circles) and a larger sample (plus signs) representing the LMC cluster system, as observed by means of integrated photometry. The lower panel includes only clusters in the present sample with colours corrected for reddening from Table 3. define the continuum according to Bica \& Alloin (1986). The EWs of $\mathrm{H}$ Balmer, KCaII, G band $(\mathrm{CH})$ and $\mathrm{MgI}$ $(5167+5173+5184) \AA$ were measured within the spectral windows defined by Bica \& Alloin (1986) and using IRAF task splot. Boundaries for the $\mathrm{KCa}$ II, G band $(\mathrm{CH}), \mathrm{MgI}, \mathrm{H} \delta, \mathrm{H} \gamma$ and $\mathrm{H} \beta$ spectral windows are, respec-

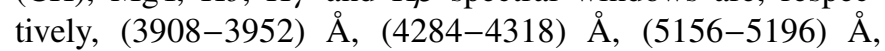

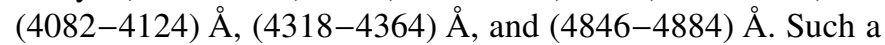
procedure has been consistently applied, making the EWs from integrated spectra safely comparable with those in the wellknown cluster database. Table 2 presents these measurements as well as the sum of EWs of the three metallic lines $\left(S_{\mathrm{m}}\right)$ and of the three Balmer lines $\mathrm{H}_{\delta}, \mathrm{H}_{\gamma}$ and $\mathrm{H}_{\beta}\left(S_{\mathrm{h}}\right)$. Typical errors of $\approx 10 \%$ on individual $\mathrm{EW}$ measurements were the result of tracing slightly different continua. If the sums of EWs $S_{\mathrm{h}}$ and $S_{\mathrm{m}}$ are separately used, the EW relative errors are improved ( $\approx 7 \%$ smaller than the individual EW errors). $S_{\mathrm{m}}$ and $S_{\mathrm{h}}$ prove to be useful in the discrimination of old, intermediate-age and young systems (Rabin 1982; Dutra et al. 1999, SP).

As a first approach to get cluster ages, Fig. 2 shows the cluster sample plotted in the diagnostic diagrams defined by SP, which are intended to discriminate cluster ages for systems younger than $10 \mathrm{Gyr}$, and metallicities for systems older than 10 Gyr. In order to show how sensitive the EW sums are on the size of the spatial profile extraction and on the stochastic effects produced by few bright stars in the integrated spectrum, we used the clusters NGC 1902 and SL 709. Two spectra were extracted from the spatial profile of NGC 1902, one of them sampling a larger cluster extent (resulting a smaller $S_{\mathrm{h}}$ ) than the other. Besides the observed integrated spectrum of SL 709, another spectrum was obtained by subtracting a bright star 
Table 2. Equivalent widths ( $)$.

\begin{tabular}{lcccccccc}
\hline \hline Feature & K Ca II & $\mathrm{H}_{\delta}$ & G band (CH) & $\mathrm{H}_{\gamma}$ & $\mathrm{H}_{\beta}$ & $\mathrm{Mg} \mathrm{I}$ & $S_{\mathrm{h}}$ & $S_{\mathrm{m}}$ \\
Windows (A) & 3908-3952 & $4082-4124$ & $4284-4318$ & $4318-4364$ & $4846-4884$ & $5156-5196$ & & \\
\hline Cluster & & & & & & & & \\
NGC 1804 & 1.9 & 7.7 & 1.1 & 8.1 & 6.0 & 1.4 & 21.8 & 4.4 \\
NGC 1839 & 4.7 & 8.6 & 1.7 & 8.7 & 7.2 & 1.1 & 24.5 & 7.5 \\
SL 237 & 0.7 & 6.5 & 1.3 & 6.4 & 4.3 & 3.4 & 17.2 & 5.4 \\
NGC 1870 & 2.5 & 7.8 & 0.2 & 7.0 & 6.6 & 1.5 & 21.4 & 4.2 \\
NGC 1894 & 4.5 & 6.8 & 1.9 & 6.2 & 4.6 & 2.5 & 17.6 & 8.9 \\
NGC 1902 & 2.9 & 7.6 & 2.0 & 8.4 & 5.9 & 2.2 & 21.9 & 7.1 \\
NGC 1902 & 1.9 & 9.0 & 2.5 & 9.6 & 6.1 & 2.7 & 24.7 & 7.1 \\
NGC 1913 & 2.4 & 5.7 & 0.8 & 5.0 & 3.9 & 2.0 & 14.6 & 5.2 \\
NGC 1932 & 6.4 & 5.2 & 2.2 & 5.5 & 4.9 & 2.2 & 15.6 & 10.8 \\
NGC 1943 & 4.7 & 8.9 & 1.3 & 7.5 & 4.0 & 2.0 & 20.4 & 8.0 \\
NGC 1940 & 3.6 & 8.2 & 0.7 & 7.9 & 6.3 & 2.2 & 22.4 & 6.5 \\
NGC 1971 & 3.3 & 8.3 & 1.1 & 8.3 & 6.3 & 1.5 & 22.9 & 5.9 \\
SL 508 & 3.4 & 8.5 & 1.1 & 7.0 & 5.5 & 2.7 & 21.0 & 7.2 \\
NGC 2038 & 1.9 & 8.3 & 0.9 & 7.8 & 5.7 & 2.1 & 21.8 & 4.9 \\
SL 709 & 3.2 & 3.9 & 3.7 & 8.8 & 5.5 & 7.0 & 18.2 & 13.9 \\
SL 709 & 3.2 & 7.1 & 3.0 & 6.9 & 6.8 & 6.2 & 20.8 & 12.4 \\
NGC 2118 & 1.3 & 8.9 & 0.8 & 8.8 & 7.2 & 1.3 & 24.9 & 3.4 \\
NGC 2130 & 1.3 & 7.4 & 2.2 & 5.1 & 5.1 & 1.7 & 17.6 & 5.2 \\
NGC 2135 & 2.8 & 9.7 & 1.9 & 9.1 & 6.6 & 1.6 & 25.4 & 6.3 \\
\hline
\end{tabular}

${ }^{a}$ Larger areal extraction than that for NGC 1902.

${ }^{b}$ Bright star subtracted from SL 709 integrated spectrum.

superimposed on the cluster bulk profile (resulting a larger $S_{\mathrm{h}}$ ). Subsequently we only used the NGC 1902 spectrum from its larger extraction, since we judged that it samples more properly the cluster population and the SL 709 spectrum with the bright star subtracted, which also should better represent the cluster bulk stellar content. The linked symbols in the diagrams correspond to two different spectral extractions from the spatial profiles of NGC 1902 and SL 709, which exemplifies the sensitivity of the integrated spectra on the size of the extraction (for NGC 1902) and on the presence of a bright star superimposed on the cluster bulk profile (for SL 709). The integrated spectra of NGC 1902 resulted in EW sums which place the cluster in the same region of the diagrams, whereas for SL 709 a transition occurs between two regions with intermediate ages. Indeed, SL 709 seems to be still further affected by an additional superimposed bright star of a younger age, which was taken into account in the analysis (see Sect. 3.2).

The clusters were then age-ranked according to the calibrations provided by SP. Since the EW of each Balmer line is a bivalued function of age with a maximum around $300 \mathrm{Myr}$, we used $S_{\mathrm{m}}$ to get a first age estimate using:

$\log t(\mathrm{Gyr})=a_{0}+a_{1} \cdot S_{\mathrm{m}}+a_{2} \cdot S_{\mathrm{m}}{ }^{2}$,

where $a_{0}=-2.18 \pm 0.38, a_{1}=0.188 \pm 0.080$ and $a_{2}=$ $-0.0030 \pm 0.0032$.

We then used $S_{\mathrm{h}}$ to get a second age estimate guided by the previous $S_{\mathrm{m}}$ estimate, since from $S_{\mathrm{h}}$ two solutions are possible:

$\log t(\mathrm{Gyr})=\frac{-b \pm \sqrt{b^{2}-4 \cdot a \cdot\left(c-S_{\mathrm{h}}\right)}}{2 \cdot a}$

where $a=-6.35 \pm 0.18, b=-8.56 \pm 0.35$ and $c=23.32 \pm 0.20$.
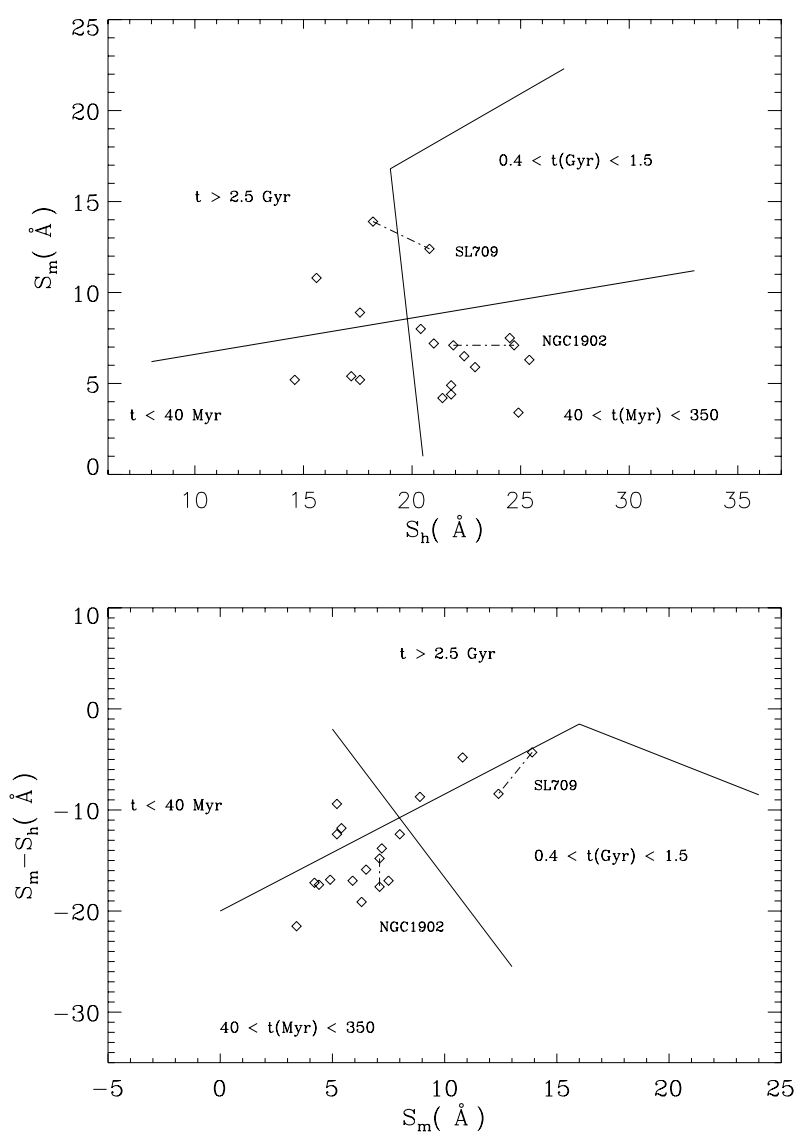

Fig. 2. Diagnostic diagrams built from EW sums of $\mathrm{H}$ Balmer and metallic lines for the 17 clusters. Continuous lines divide regions of different age ranges. The corresponding EW sums for both spectra of SL 709 and NGC 1902 are linked. 
Table 3. Cluster parameters.

\begin{tabular}{|c|c|c|c|c|c|c|}
\hline Cluster & $E(B-V)$ & $\begin{array}{c}t_{\text {literature }} \\
(\mathrm{Gyr})\end{array}$ & Ref. & $\begin{array}{l}t_{\mathrm{Sh}, \mathrm{Sm}} \\
(\mathrm{Gyr})\end{array}$ & $\begin{array}{c}t_{\text {template }} \\
(\mathrm{Gyr})\end{array}$ & $\begin{array}{l}t_{\text {adopted }} \\
\text { (Gyr) }\end{array}$ \\
\hline NGC 1804 & 0.08 & $0.08 \pm 0.01$ & 1 & $0.035 \pm 0.004$ & $0.05 \pm 0.01$ & $0.06 \pm 0.02$ \\
\hline \multirow[t]{3}{*}{ NGC 1839} & 0.06 & $0.10 \pm 0.01$ & 1 & $0.09 \pm 0.02$ & 0.06 & $0.09 \pm 0.03$ \\
\hline & & $0.033 \pm 0.008$ & 2 & & & \\
\hline & & $0.125 \pm 0.025$ & 4 & & & \\
\hline \multirow[t]{2}{*}{ SL 237} & 0.07 & $0.038 \pm 0.004$ & 1 & $0.04 \pm 0.02$ & $0.05 \pm 0.01$ & $0.04 \pm 0.02$ \\
\hline & & $0.027 \pm 0.009$ & 2 & & & \\
\hline \multirow[t]{2}{*}{ NGC 1870} & 0.08 & $0.09 \pm 0.01$ & 1 & $0.033 \pm 0.004$ & $0.05 \pm 0.01$ & $0.06 \pm 0.03$ \\
\hline & & $0.07 \pm 0.03$ & 2 & & & \\
\hline NGC 1894 & 0.09 & $0.071 \pm 0.008$ & 1 & $0.10 \pm 0.08$ & $0.13 \pm 0.03$ & $0.10 \pm 0.03$ \\
\hline NGC 1902 & 0.04 & - & & $0.07 \pm 0.03$ & 0.06 & $0.07 \pm 0.03$ \\
\hline NGC 1913 & 0.09 & $0.024 \pm 0.002$ & 1 & $0.03 \pm 0.02$ & 0.06 & $0.04 \pm 0.02$ \\
\hline NGC 1932 & 0.05 & - & & $0.2 \pm 0.2$ & $0.4 \pm 0.2$ & $0.3 \pm 0.2$ \\
\hline \multirow[t]{2}{*}{ NGC 1943} & 0.08 & $0.14 \pm 0.02$ & 1 & $0.08 \pm 0.06$ & $0.28 \pm 0.08$ & $0.14 \pm 0.06$ \\
\hline & & $0.10 \pm 0.01$ & 3 & & & \\
\hline NGC 1940 & 0.06 & - & & $0.06 \pm 0.02$ & 0.06 & $0.06 \pm 0.02$ \\
\hline NGC 1971 & 0.06 & $0.10 \pm 0.01$ & 1 & $0.05 \pm 0.01$ & $0.05 \pm 0.01$ & $0.06 \pm 0.02$ \\
\hline SL 508 & 0.06 & $0.10 \pm 0.01$ & 1 & $0.07 \pm 0.04$ & 0.06 & $0.07 \pm 0.04$ \\
\hline NGC 2038 & 0.06 & $0.13 \pm 0.02$ & 1 & $0.039 \pm 0.008$ & 0.06 & $0.08 \pm 0.05$ \\
\hline SL 709 & 0.06 & - & & $0.3 \pm 0.2$ & $0.13 \pm 0.03$ & $0.3 \pm 0.2$ \\
\hline NGC 2118 & 0.07 & - & & $0.05 \pm 0.02$ & 0.06 & $0.05 \pm 0.02$ \\
\hline NGC 2130 & 0.05 & - & & $0.03 \pm 0.02$ & 0.06 & $0.04 \pm 0.02$ \\
\hline NGC 2135 & 0.05 & - & & $0.085 \pm 0.008$ & $0.05 \pm 0.01$ & $0.07 \pm 0.02$ \\
\hline
\end{tabular}

References: (1) Pietrzyński \& Udalski (2000); (2) Alcaino \& Liller (1987); (3) Bono et al. (2005); (4) Piatti et al. (2003a).

The average of these two estimates is listed in Col. 5 of Table 3.

\subsection{Template matching method}

The template matching method consists in achieving the best possible match between the analyzed cluster spectrum and a template spectrum of known age and metallicity. In this process we selected, from the available template spectra, the ones which minimize the flux residuals, calculated as the normalized difference (cluster - template)/cluster. Note that differences between cluster and template spectra are expected to be found due to variations in the stellar composition of the cluster, such as the presence of a relatively bright star with particular spectral features or contamination of a field star close to the direction towards the cluster.

All 17 clusters in our sample are well represented by blue stellar populations, according to their spectral properties. For the present sample, the useful template spectra are: Yd (40 Myr), Ye (45-75 Myr), Yf (100-150 Myr), Yg (200-350 Myr) and Yh (0.5 Gyr), which represent young and intermediate-age populations built from Galactic open clusters (Piatti et al. 2002b).

Since the continuum distribution is also affected by reddening, we firstly adopted a colour excess $E(B-V)$ for each cluster, taking into account the Burstein \& Heiles (1982, hereafter BH) extinction maps. Secondly, we corrected the observed spectra accordingly and then we applied the template match method. The results are shown in Figs. 6 to 22 .

Three clusters (SL 237, SL 508 and SL 709) have their spectra affected by a bright star, which was taken into account according to the following procedure: we matched the spectrum of SL 237 with a combination of templates Yd (40 Myr) and Ye (60 Myr) and an average spectrum of giant stars of early M type from Silva \& Cornell (1992). The stellar spectrum contributes with $35 \%$ of the total light at $5870 \AA$ (see Fig. 20). As for SL 237, the spectrum of SL 508 shows bands redwards of $5000 \AA$, characteristic of late spectral types. The same stellar spectrum used in the spectral matching of SL 237 was also used for SL 508 in combination with template Ye. The star contributes with $15 \%$ of the integrated light at $5870 \AA$ (see Fig. 21). The same procedure was applied to SL 709, which presents a flat continuum beyond $5000 \AA$. In the case of SL 709, the spectrum of the Carbon star TT Tau (Barnbaum et al. 1996), with $20 \%$ of contribution to the total flux at $5870 \AA$, was combined with template Yf (see Fig. 22).

\section{Adopted ages}

The ages determined by the two methods, together with estimates from the literature (whenever available), were used to 


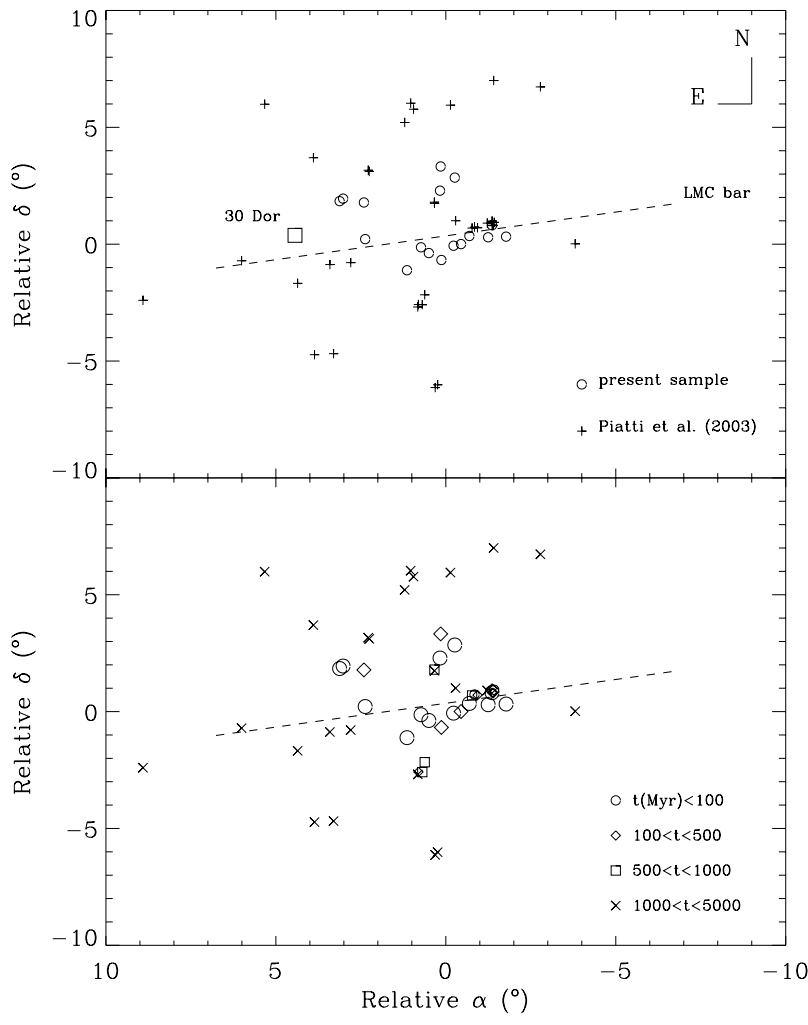

Fig. 3. Spatial distribution of LMC clusters with ages in a homogeneous scale. Upper panel: the present sample and the sample analyzed in Piatti et al. (2003b) are plotted in equatorial coordinates relative to the LMC center. The positions of the bar and 30 Dor are indicated. Lower panel: the same data is plotted with different symbols discriminating different age ranges.

get final averaged ages (Table 3). Their respective errors take into consideration the dispersion of the values averaged. The reddening adopted in the template match method is presented in Col. 2 of Table 3. The LMC is optically thin, the average foreground and internal $E(B-V)$ colour excesses being 0.06 and $0.06 \mathrm{mag}$, respectively (Dutra et al. 2001). This explains why the derived $E(B-V)$ values turn out to be relatively small, being all lower than $0.1 \mathrm{mag}$.

Ten of the clusters in our sample are also present in OGLEII sample (Pietrzyński et al. 1999). Pietrzyński \& Udalski (2000) determined their ages by fitting isochrones of $Z=0.008$ on cluster colour-magnitude diagrams (CMDs) built with OGLEII data (see Table 3). By analyzing the distribution in the BV photographic CMD of cluster member stars, Alcaino \& Liller (1987) derived age for NGC 1839, NGC 1870 and SL 237 considering the position of the main sequence turnoff, the position of the brightest blue star and the fitting of Maeder \& Mermilliod (1981) isochrones. Their results are displayed in Table 3. Bono et al. (2005) determined the age of NGC 1943 by analyzing the pulsational period of its 9 Cepheids and found it consistent with the value obtained from isochrone fitting on OGLEII data. The value shown in Table 3 is an average of their results.

Within the expected uncertainties, the ages derived in the present work agree with those given in the literature.

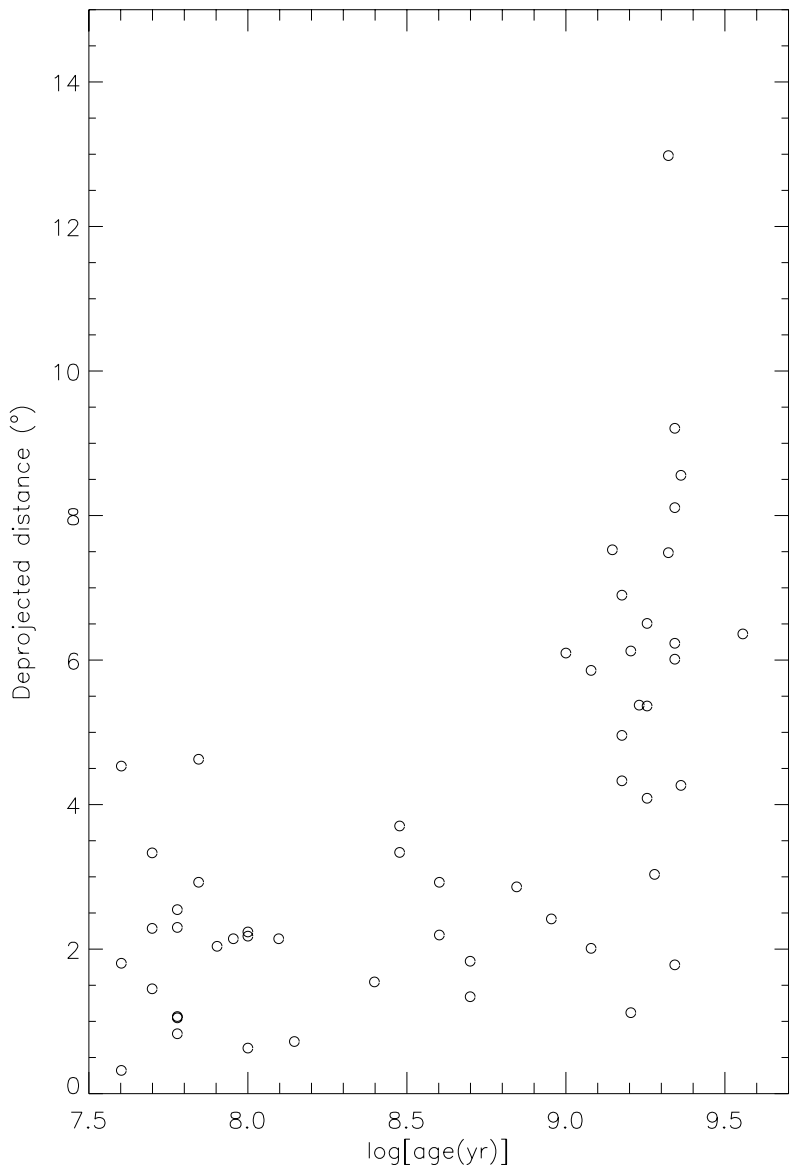

Fig. 4. Deprojected distance from the LMC center (in degrees) as a function of age for the same sample of Fig. 3 .

\section{Age vs. spatial distribution}

Piatti et al. (2003b) carried out observations in the Washington photometric system for 6 LMC clusters, which increased up to 37 the total sample of young and intermediate age clusters with uniform estimates of age and metallicity. These parameters were determined using CMDs and theoretical isochrones. Figure 3 shows the spatial projected distribution of the clusters in our sample and the sample of 37 clusters previously studied (upper panel). Right ascension and declination are relative to the LMC center, considered as the position of the cluster NGC $1928\left(\alpha(\mathrm{J} 2000)=5 \mathrm{~h} 20 \mathrm{~m} 57 \mathrm{~s}, \delta(\mathrm{J} 2000)=-69^{\circ} 28^{\prime} 41^{\prime \prime}\right)$. The lower panel of this figure discriminates clusters of different ages in the whole sample of 53 clusters (NGC 1839 is in both samples). It can be noticed that the general tendency is for the older clusters to lie in the outer disk regions of the galaxy while the younger ones tend to be located not far from or in the bar. This effect is easily observed when the deprojected galactocentric distance is plotted against age, as shown in Fig. 4. The deprojected galactocentric distance was calculated using an inclination between the outer LMC disk and the plane of the sky of $45^{\circ}$ and a position angle of the line of nodes of $7^{\circ}$ (Lyngå \& Westerlund 1963). This tendency is compatible with the findings of Smecker-Hane et al. (2002) who derived the LMC star formation history from HST observations of field stars. They present a detailed comparative analysis of CMDs of 

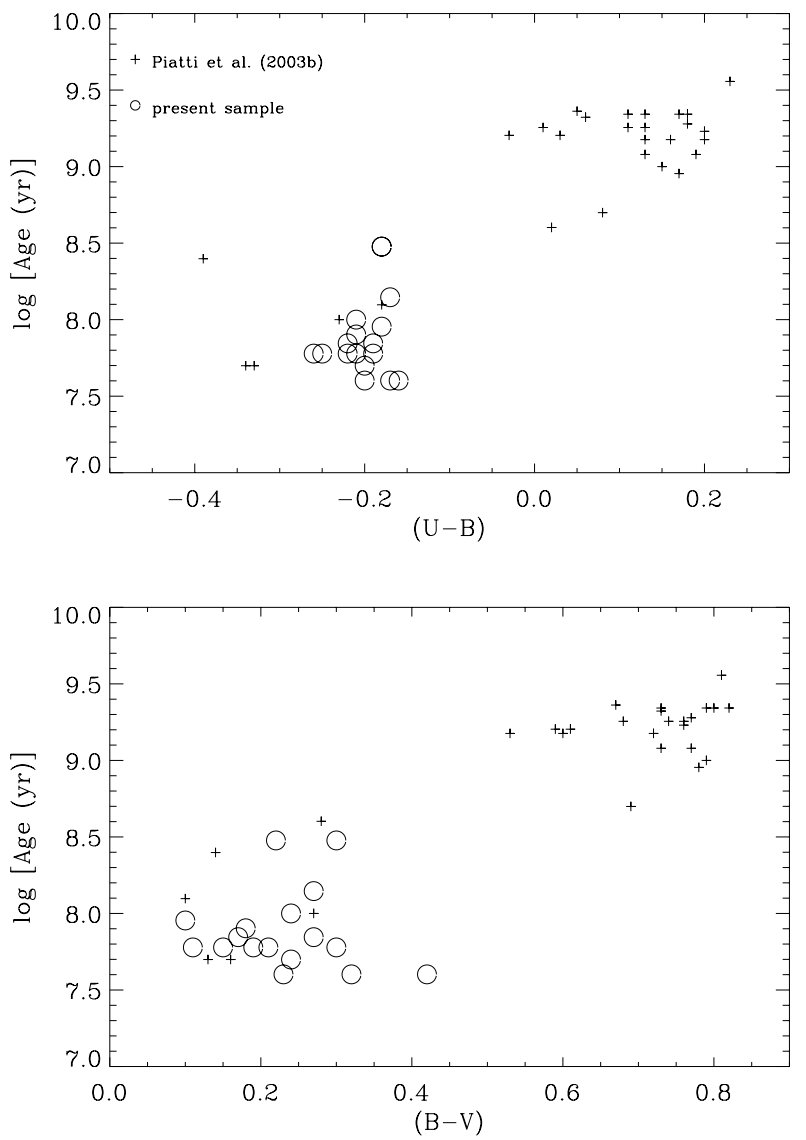

Fig. 5. Distribution of cluster age with integrated colours for the 17 clusters plus the sample used by Piatti et al. (2003b).

the bar and the disk, concluding that the star formation history is different in these regions for ages $<6 \mathrm{Gyr}$. The star formation rate seems to have increased around $\approx 2$ Gyr ago for both disk and bar, though it has declined more recently in the disk while remaining roughly constant in the bar (Smecker-Hane et al. 2002). However, cluster formation does not seem to follow star formation in their detailed histories.

\section{Age vs. integrated colours}

Integrated $U B V$ colours of $624 \mathrm{LMC}$ associations and clusters were obtained by Bica et al. (1996) from photoelectric photometry observations. We used these data to check the trend of $(U-B)$ and $(B-V)$ with age according to the present estimates. For clusters with integrated photometry, Fig. 5 shows this comparison for the present sample and for the one presented in Piatti et al. (2003b), totalling 53 clusters with uniform age estimates. The colour gap seen in both $(U-B)$ and $(B-V)$ is a real feature first identified by van den Bergh (1981) and supported by larger cluster samples (Bica et al. 1996). The gap is probably a natural consequence of cluster evolution with increasing metallicities towards the present, and epochs of reduced cluster formation between $\approx 300 \mathrm{Myr}$ and $\approx 1 \mathrm{Gyr}$ (e.g. Girardi et al. 1995). At least in the LMC bar, such a period of reduced cluster formation is not observed for the field stars (Smecker-Hane et al. 2002).

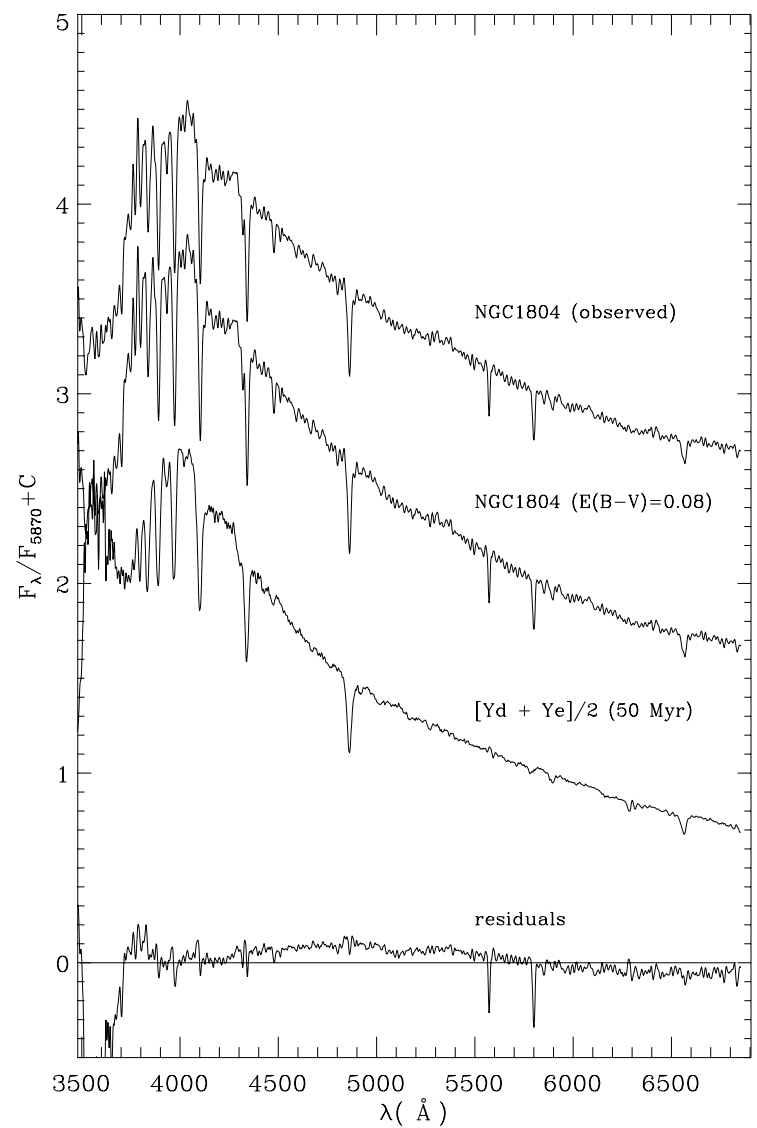

Fig. 6. From top to bottom: the observed integrated spectrum of NGC 1804, the spectrum corrected for the adopted reddening $E(B-V)$, the template spectrum which best matches it, and the flux residuals according to $\left(F_{\text {cluster }}-F_{\text {template }}\right) / F_{\text {cluster }}$.

\section{Conclusions}

We have determined ages of blue LMC star clusters using their integrated spectra. The results obtained from two independent methods are in agreement with the literature estimates for clusters studied in common.

The present sample, added to a set of young and intermediate-age clusters with uniformly determined ages, was used with the aim of analyzing their spatial distribution. Clusters closer to the LMC bar tend to be younger than those at the LMC disk. The colour gap at $(U-B) \approx-0.1$ and $(B-V) \approx 0.5$ noticed in studies with larger cluster samples appears to be related to the natural cluster evolution, as suggested by the present analysis. However, the older groups SWB III, SWB IVa and SWB IVb must be observed spectroscopically and in detail by means of CMDs, testing also the possibility of decreased cluster formation contributing to the gap.

Acknowledgements. We would like to thank the CASLEO staff members and technicians for their kind hospitality and support during the observing runs. We are grateful for the use of the CCD and data acquisition system at CASLEO, supported under US National Science Foundation (NSF) grant AST-90-15827. We also thank the anonymous referee for helpful remarks. This work was partially supported by the Brazilian institutions CNPq and FAPEMIG, and the Argentinian institutions CONICET, Agencia Nacional de Promoción Científica y Tecnológica (ANPCyT) and Agencia Córdoba Ciencia. 


\section{References}

Ahumada, A. V., Clariá, J. J., Bica, E., \& Dutra, C. M. 2002, A\&A, 393, 855

Alcaino, G., \& Liller, W. 1987, AJ, 94, 372

Barnbaum, C., Stone, R. P. S., \& Keenan, P. C. 1996, ApJS, 105, 419

Bhatia, R. K., \& MacGillivray, H. T. 1989, A\&A, 211, 9

Bhatia, R. K., Read, M. A., Hatzidimitriou, D., \& Tritton, S. 1991, A\&AS, 87, 335

Bica, E., \& Alloin, D. 1986, A\&A, 162, 21

Bica, E., Alloin, D., \& Santos Jr., J. F. C. 1990, A\&A, 235, 103

Bica, E., Clariá, J. J., Dottori, H., Santos Jr., J. F. C., \& Piatti, A. E. 1996, ApJS, 102, 57

Bica, E., Schmitt, H. R., Dutra, C. M., \& Oliveira, H. L. 1999, AJ, 117,238

Bono, G., Marconi, M., Cassisi, S., et al. 2005, ApJ, 621, 966

Burstein, D., \& Heiles, C. 1982, AJ, 87, 1165

Dutra, C. M., Bica, E., Clariá, J. J., \& Piatti, A. E. 1999, MNRAS, 305, 373

Dutra, C. M., Bica, E., Clariá, J. J., Piatti, A. E., \& Ahumada, A. V. 2001, A\&A, 371, 895

Girardi, L., Chiosi, C., Bertelli, G., \& Bressan, A. 1995, A\&A, 298, 87

Hodge, P. 1988, PASP, 100, 1051

Kontizas, M., Morgan, D. H., Hatzidimitriou, D., \& Kontizas, E. 1990, A\&AS, 84, 527

Lauberts, A. 1982, The ESO/Uppsala Survey of the ESO (B) Atlas, European Southern Observatory, Garching bei Munchen

Lyngå, G., \& Westerlund, B. E. 1963, MNRAS, 127, 31

Maeder, A., \& Mermilliod, J.-C. 1981, A\&A, 93, 136
Mighell, K. J., Sarajedini, A., \& French, R. S. 1998, AJ, 116, 2395

Piatti, A. E., Santos Jr., J. F. C., Clariá, J. J., et al. 2001, MNRAS, 325, 792

Piatti, A. E., Sarajedini, A., Geisler, D., et al. 2002a, MNRAS, 329, 556

Piatti, A. E., Bica, E., Clariá, J. J., Santos Jr., J. F. C., \& Ahumada, A. V. 2002b, MNRAS, 335, 233

Piatti, A. E., Geisler, D., Bica, E., \& Clariá, J. J. 2003a, MNRAS, 343, 851

Piatti, A. E., Bica, E., Geisler, D., \& Clariá, J. J. 2003b, MNRAS, 344, 965

Piatti, A. E., Santos Jr., J. F. C., Clariá, J. J., et al. 2005, A\&A 440, 111

Pietrzyński, G., Udalski, A., Kubiak, M., et al. 1999, Acta Astron., 49, 521

Pietrzyński, G., \& Udalski, A. 2000, AcA, 50, 337

Rabin, D. 1982, ApJ, 261, 85

Santos Jr., J. F. C., \& Bica, E. 1993, MNRAS, 260, 915

Santos Jr., J. F. C., \& Piatti, A. E. 2004, A\&A, 428, 79 (SP)

Santos Jr., J. F. C., Bica, E., Clariá, J. J., et al. 1995, MNRAS, 276, 1155

Santos Jr., J. F. C., Alloin, D., Bica, E., \& Bonatto, C. 2002, in IAU Symp. 207, ed. D. Geisler, E. K. Grebel, \& D. Minniti (San Francisco: ASP), 727

Searle, L., Wilkinson, A., \& Bagnuolo, W. G. 1980, ApJ, 239, 803

Shapley, H., \& Lindsay, E. M. 1963, Irish Astron. J., 6, 74

Silva, D. R., \& Cornell, M. E. 1992, ApJS, 81, 865

Smecker-Hane, T. A., Cole, A. A., Gallagher, J. S., \& Stetson, P. B. 2002, ApJ, 566, 239

van den Bergh, S. 1981, A\&AS, 46,79 


\section{Online Material}


J. F. C. Santos Jr. et al.: Spectral evolution of LMC clusters, Online Material p 2
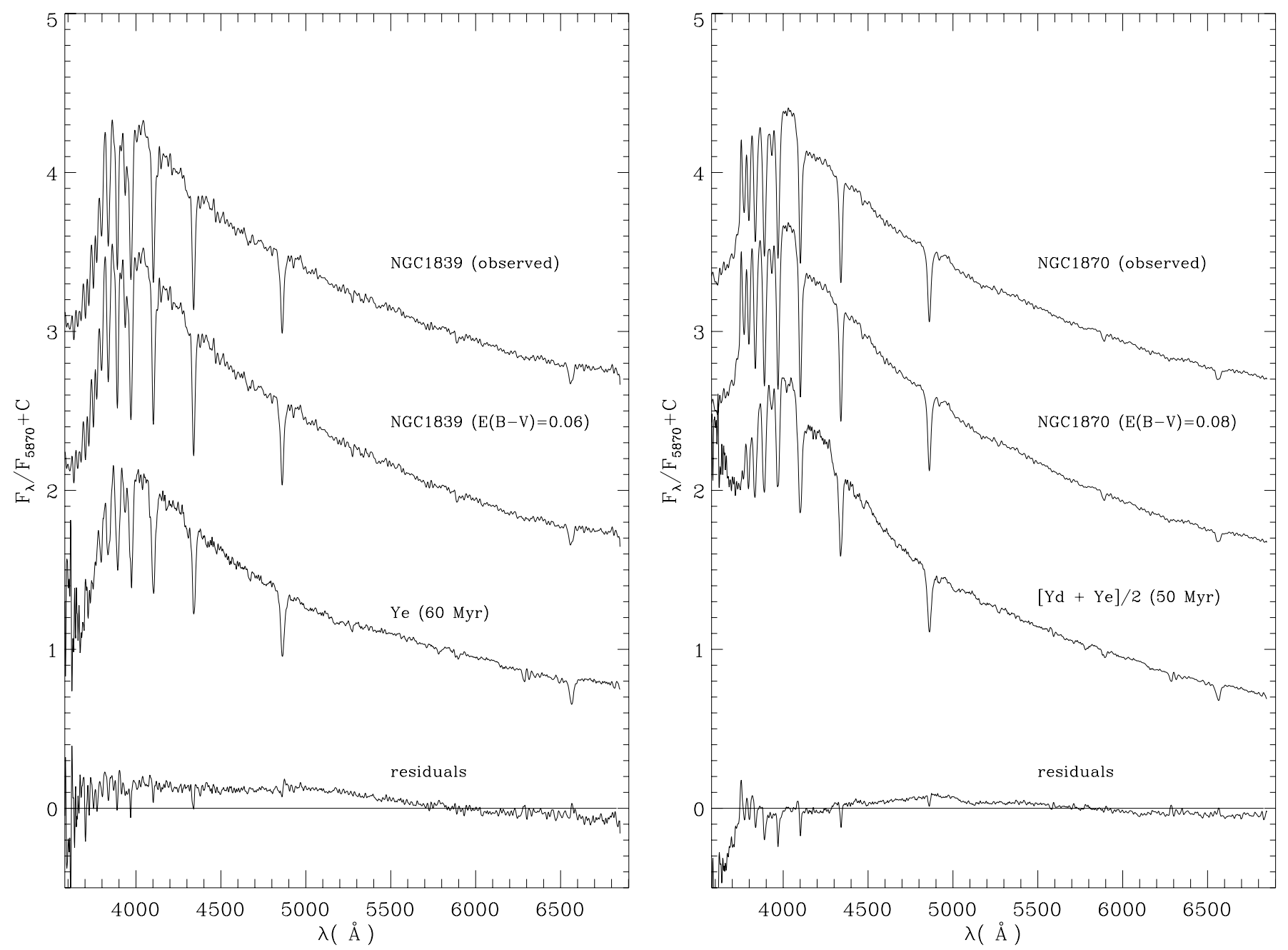

Fig. 7. Same as Fig. 6 for NGC 1839.

Fig. 8. Same as Fig. 6 for NGC 1870. 
J. F. C. Santos Jr. et al.: Spectral evolution of LMC clusters, Online Material p 3
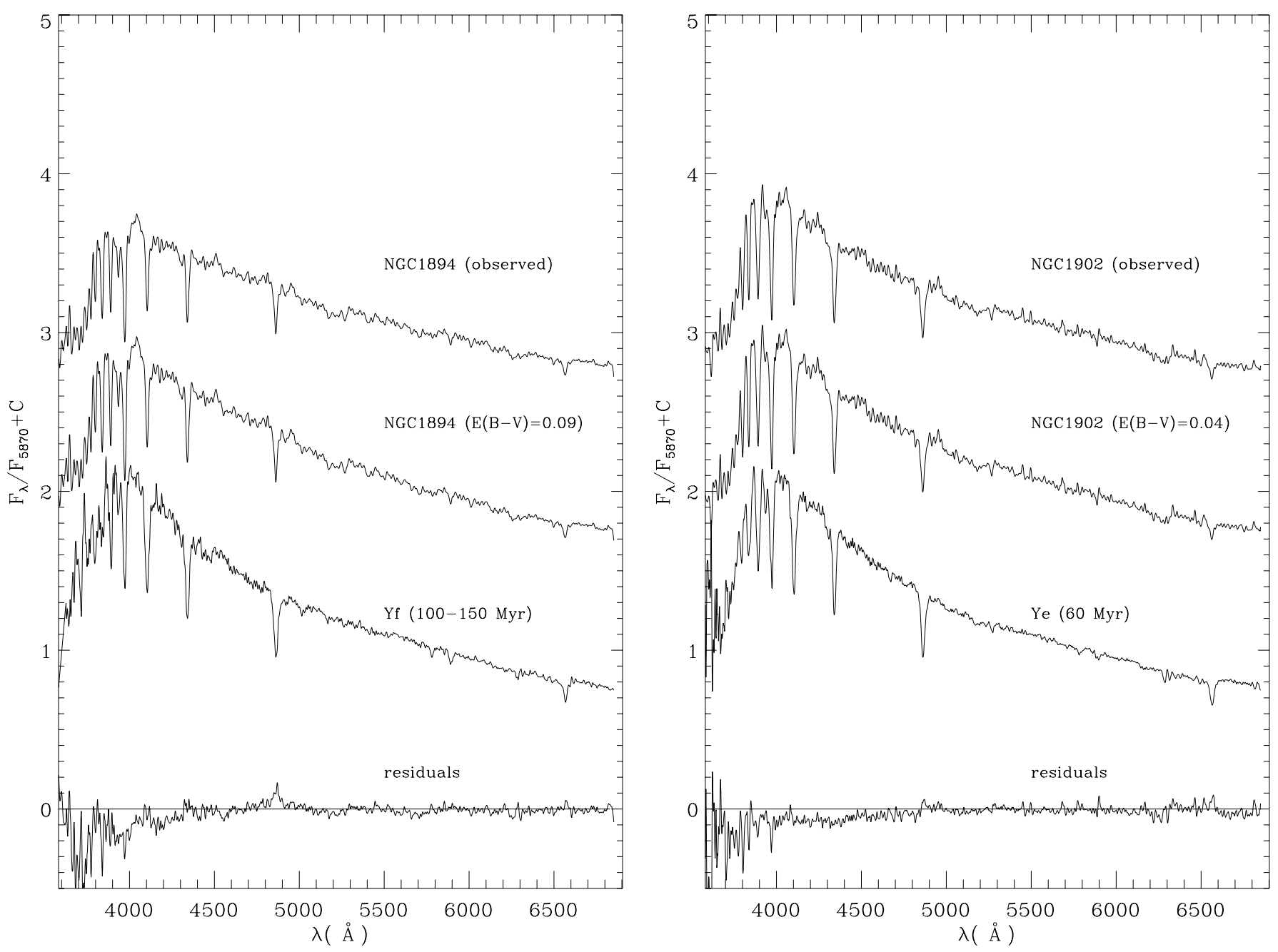

Fig. 9. Same as Fig. 6 for NGC 1894.

Fig. 10. Same as Fig. 6 for NGC 1902 (larger extraction). 
J. F. C. Santos Jr. et al.: Spectral evolution of LMC clusters, Online Material p 4
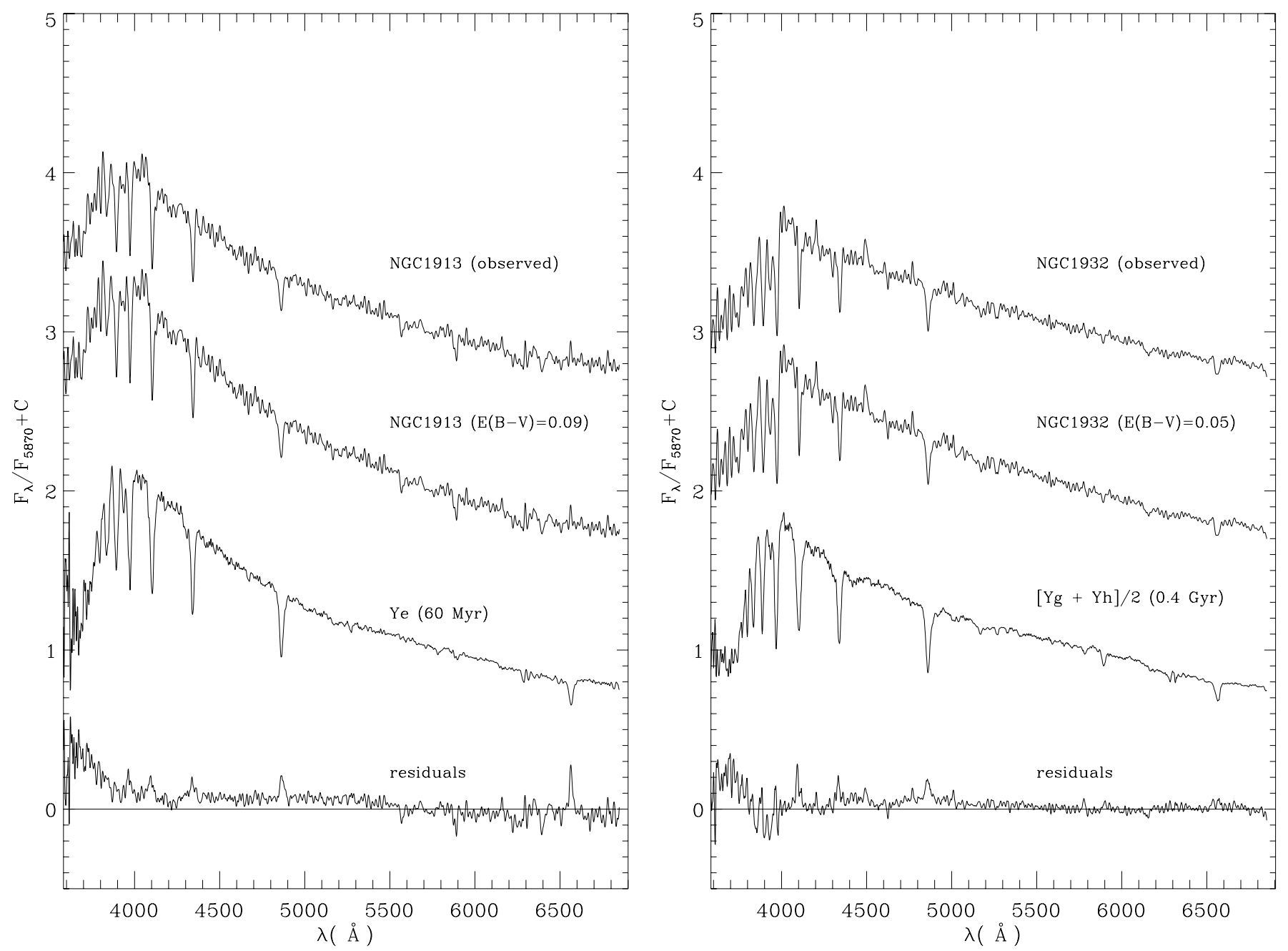

Fig. 11. Same as Fig. 6 for NGC 1913.

Fig. 12. Same as Fig. 6 for NGC 1932. 
J. F. C. Santos Jr. et al.: Spectral evolution of LMC clusters, Online Material p 5
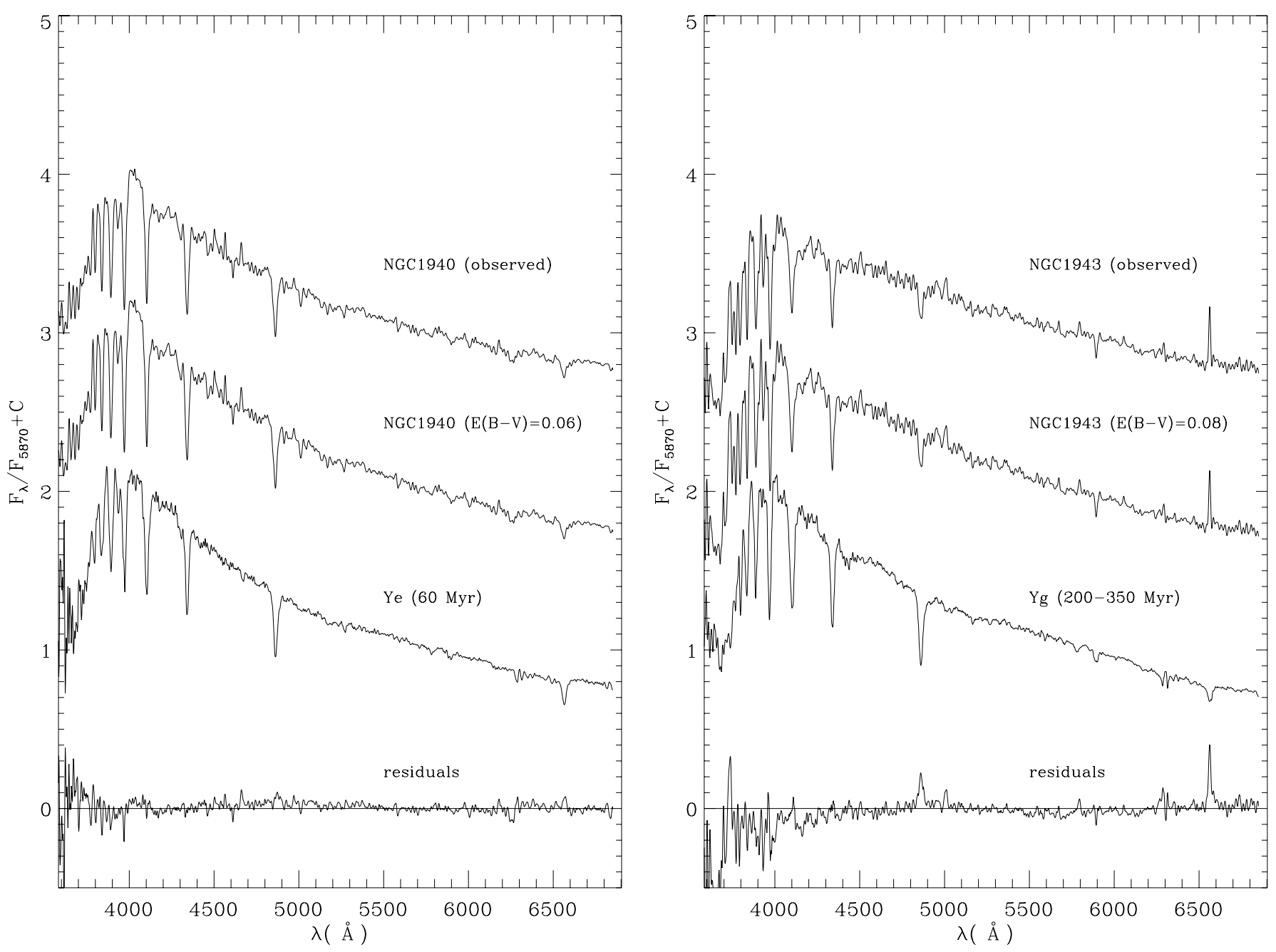

Fig. 13. Same as Fig. 6 for NGC 1940.

Fig. 14. Same as Fig. 6 for NGC 1943. 
J. F. C. Santos Jr. et al.: Spectral evolution of LMC clusters, Online Material p 6
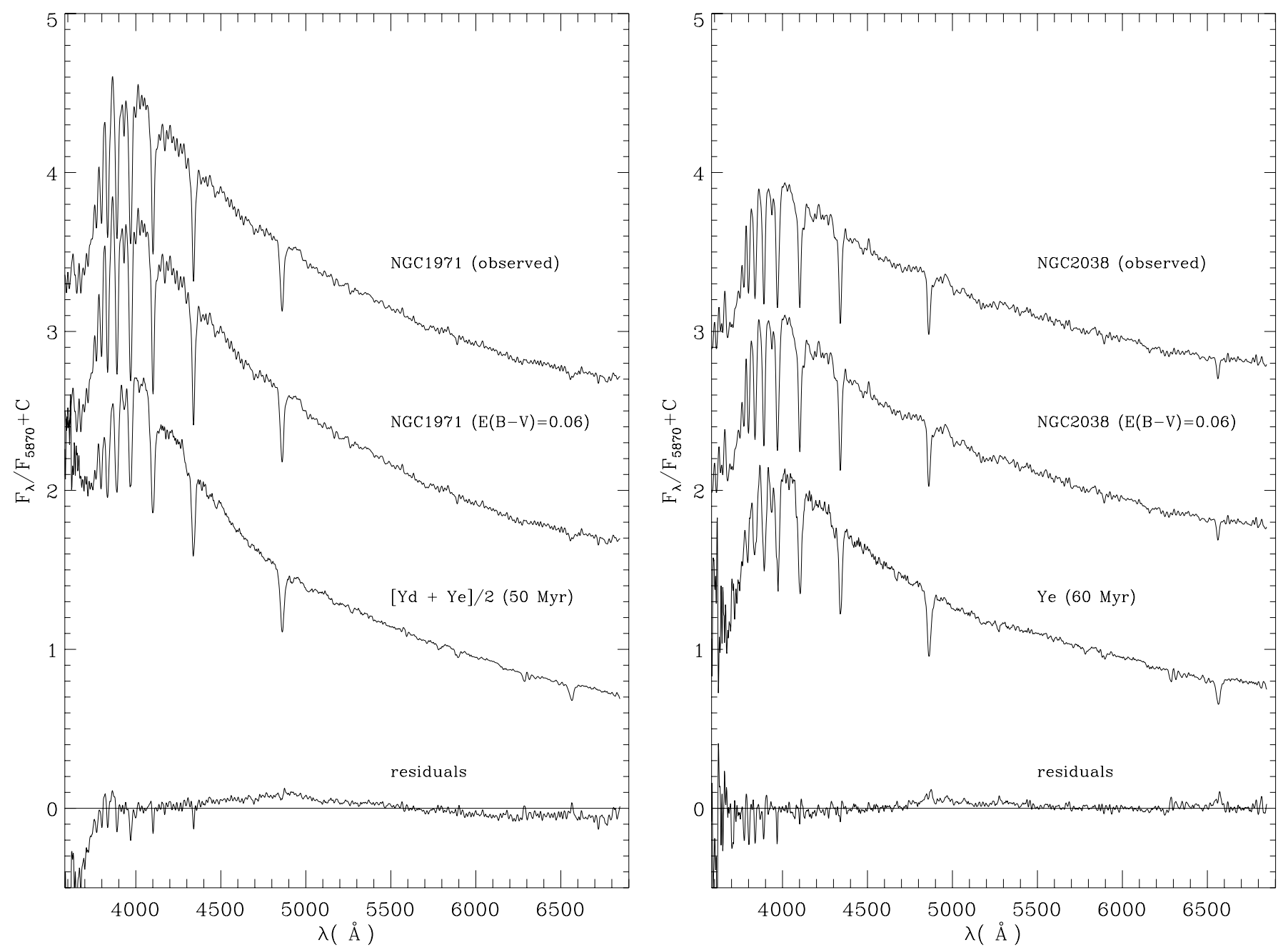

Fig. 15. Same as Fig. 6 for NGC 1971.

Fig. 16. Same as Fig. 6 for NGC 2038. 
J. F. C. Santos Jr. et al.: Spectral evolution of LMC clusters, Online Material $p 7$
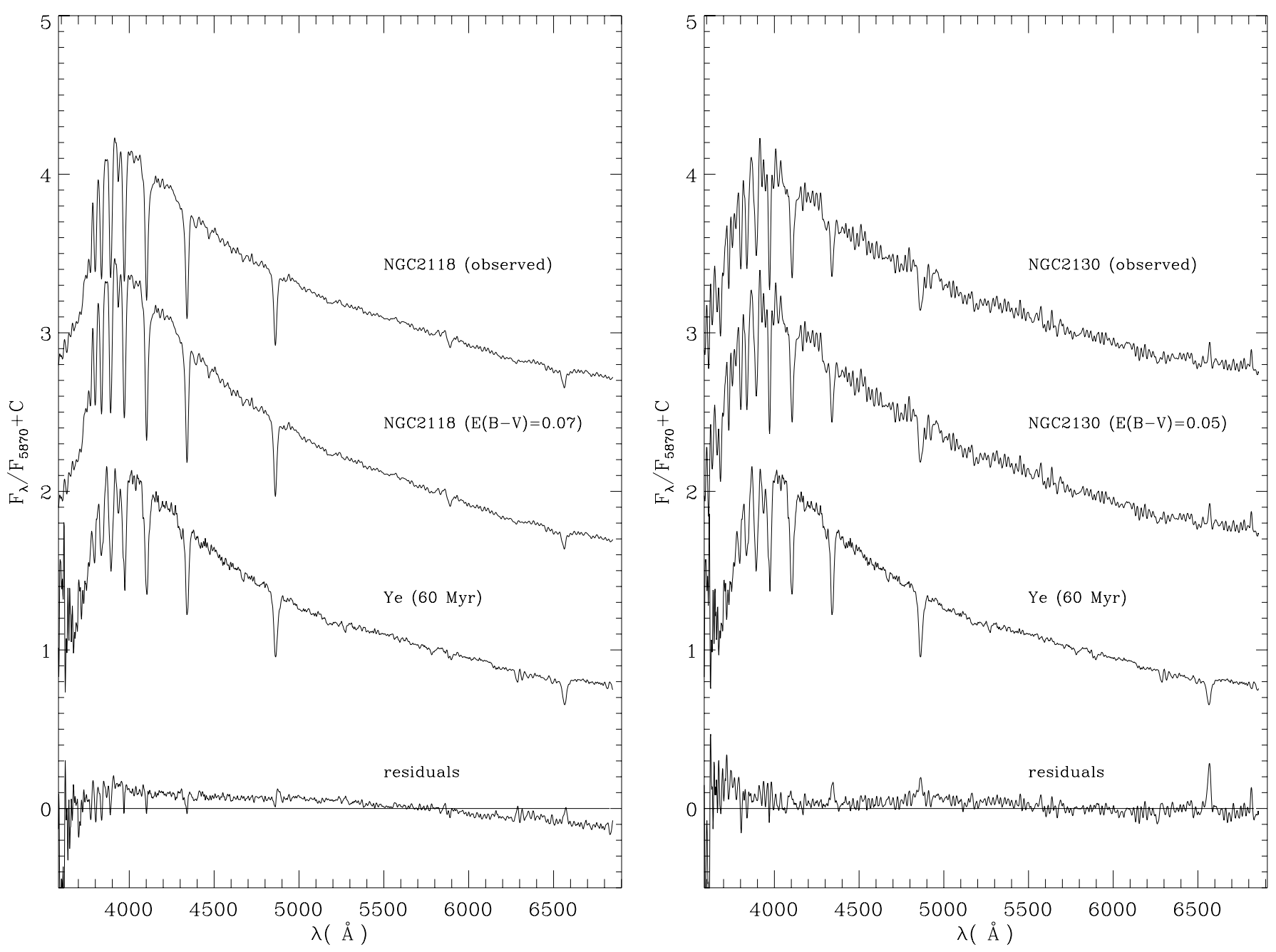

Fig. 17. Same as Fig. 6 for NGC 2118.

Fig. 18. Same as Fig. 6 for NGC 2130. 
J. F. C. Santos Jr. et al.: Spectral evolution of LMC clusters, Online Material $p 8$

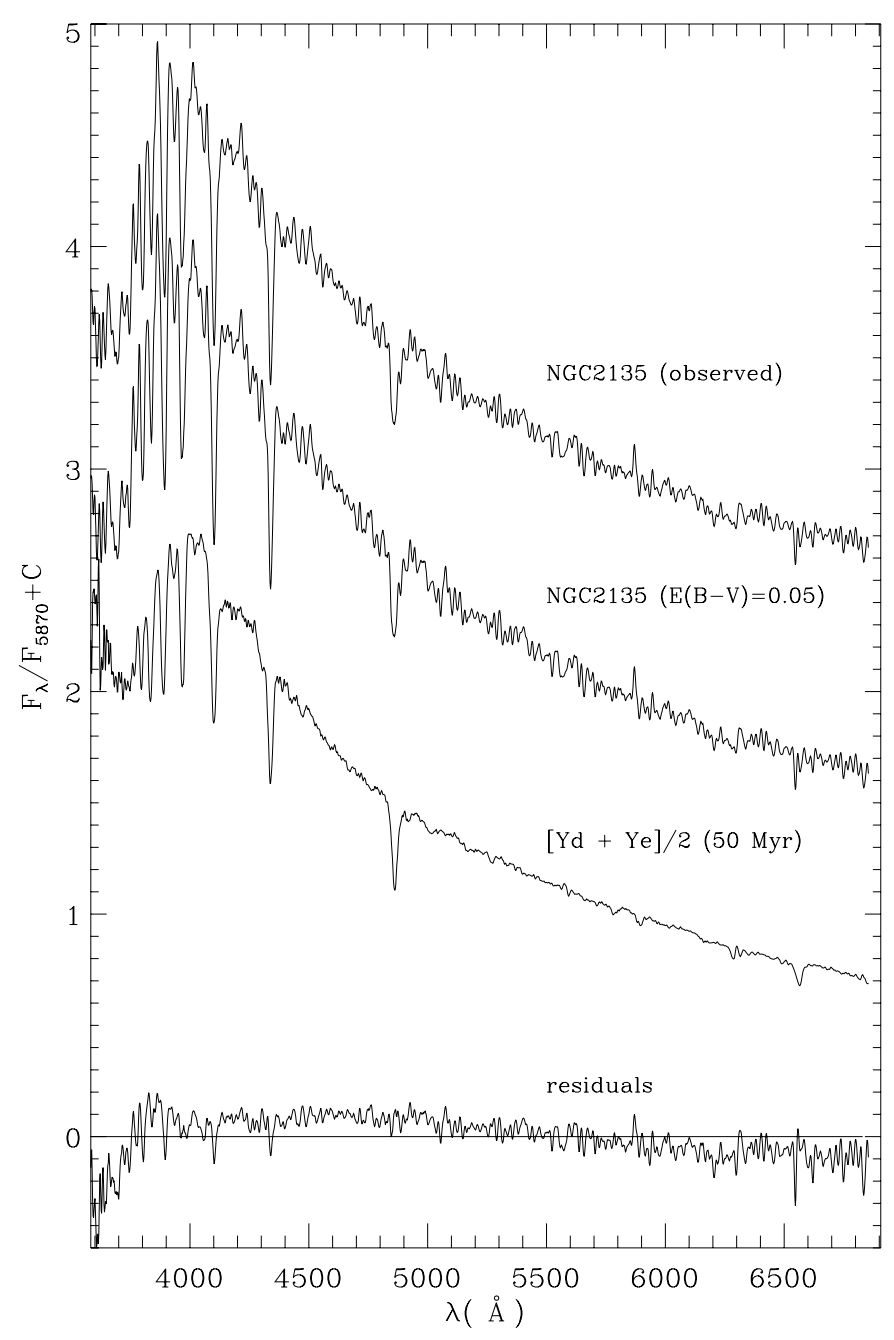

Fig. 19. Same as Fig. 6 for NGC 2135.

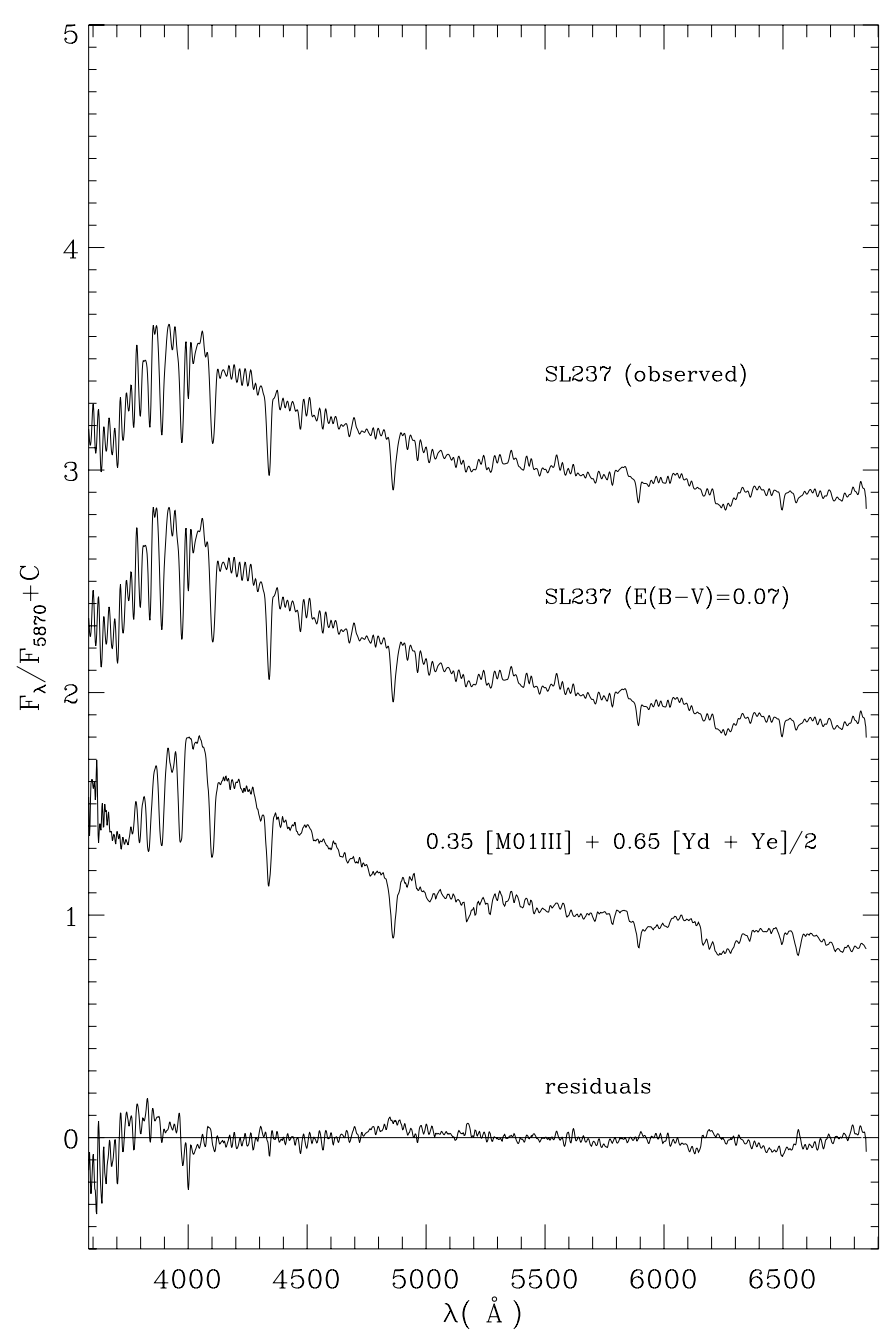

Fig. 20. Same as Fig. 6 for SL 237. The matched spectrum is a combination of templates $\mathrm{Ye}(40 \mathrm{Myr})$ and $\mathrm{Yd}(60 \mathrm{Myr})$ and an average spectrum of early M giants (Silva \& Cornell 1992). The flux fraction at $5870 \AA$ is indicated. 
J. F. C. Santos Jr. et al.: Spectral evolution of LMC clusters, Online Material $p 9$

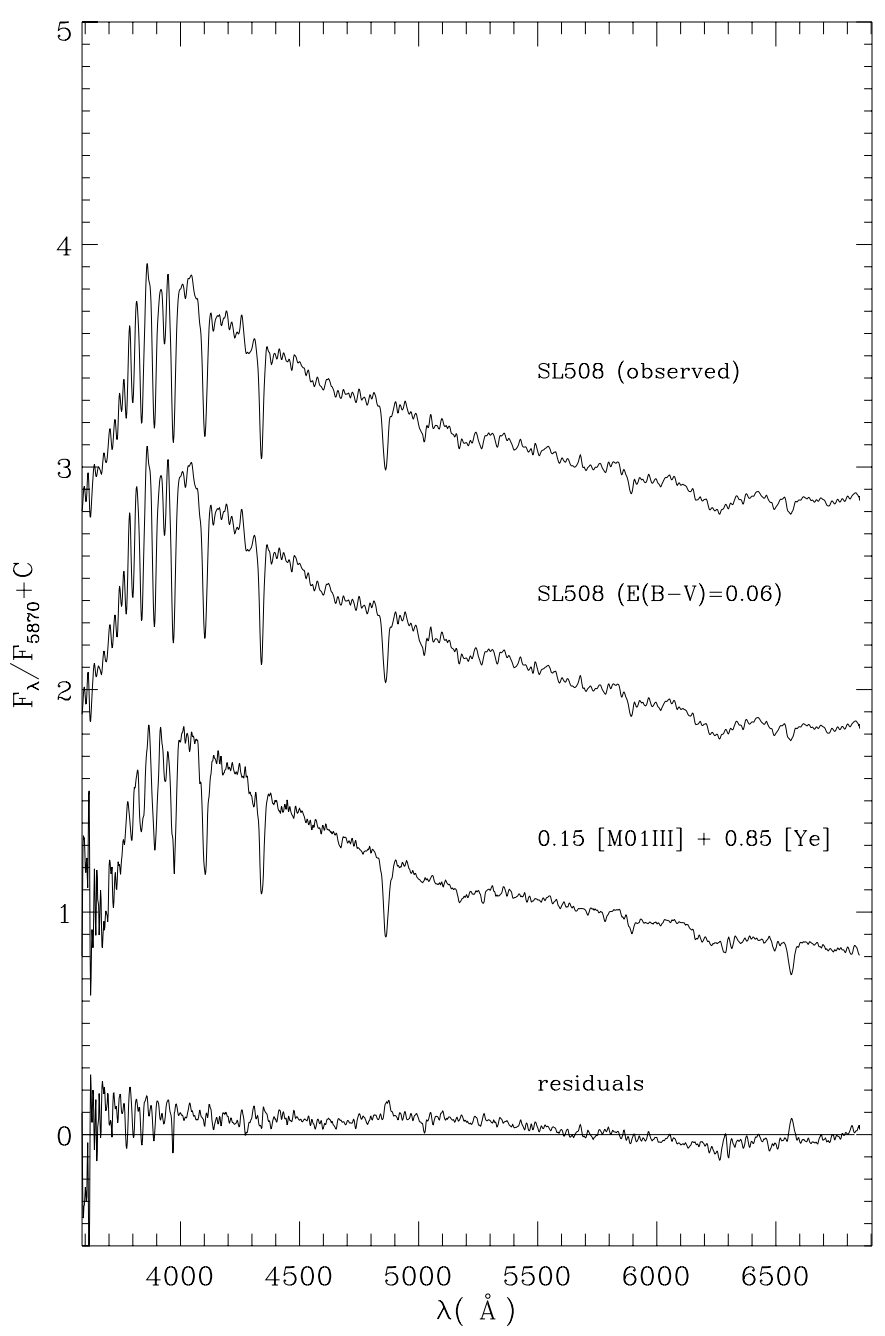

Fig. 21. Same as Fig. 6 for SL 508. The matched spectrum is a combination of template $\mathrm{Ye}(40 \mathrm{Myr})$ and an average spectrum of early $\mathrm{M}$ giants (Silva \& Cornell 1992). The flux fraction at $5870 \AA ̊$ is indicated.

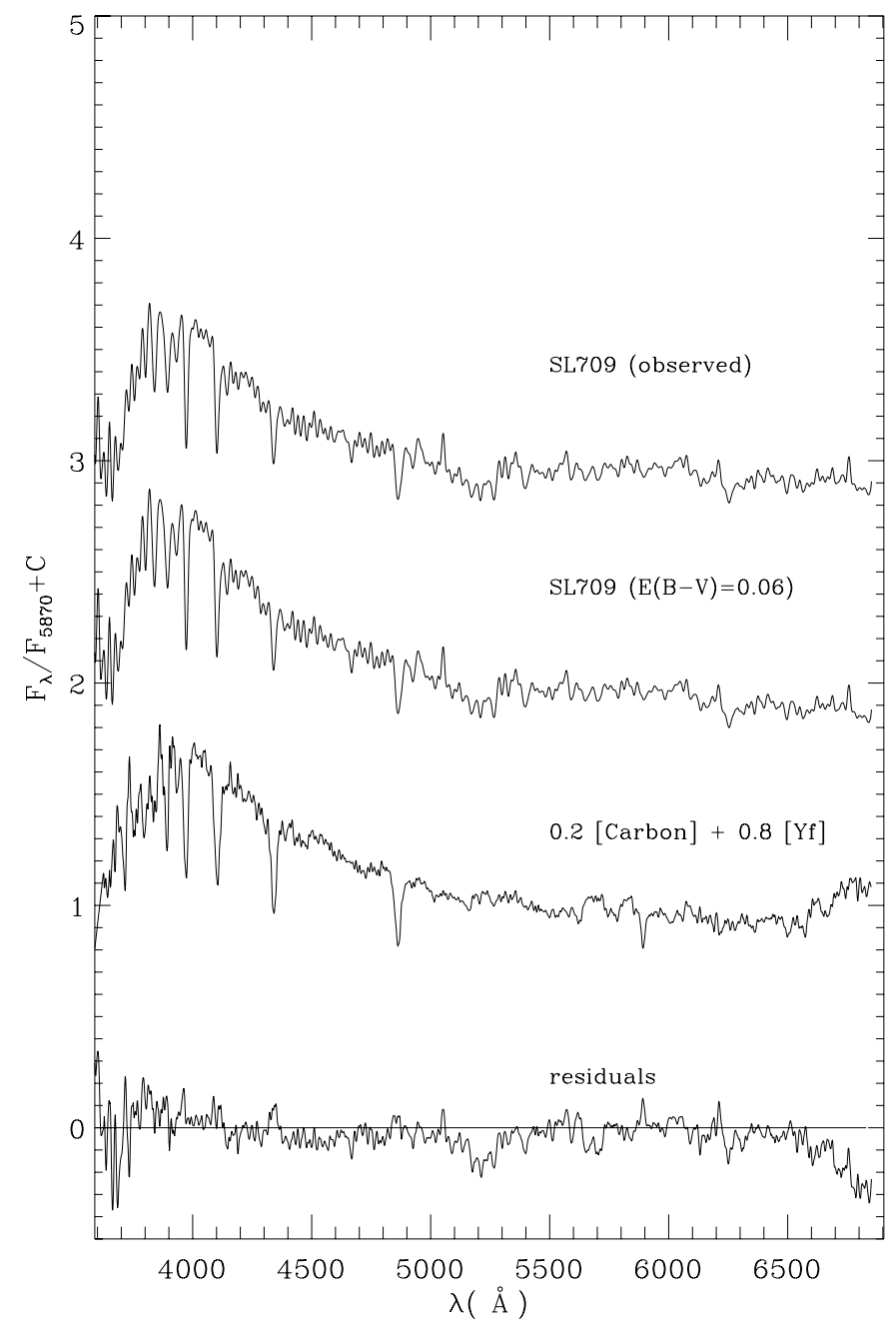

Fig. 22. Same as Fig. 6 for SL 709. The matched spectrum is a combination of template Yf (100-150 Myr) and the spectrum of a Carbon star (TT Tau from Barnbaum et al. (1996)). The flux fraction at $5870 \AA$ is indicated. 